\title{
Caracterización del Costo de Distribución de Energía Eléctrica Mediante Modelos de Fronteras de Eficiencia considerando un Indicador de Calidad del Servicio
}

\author{
Carlos A. García-Montoya( ${ }^{(1)}$, Jesús M. López-Lezama ${ }^{(2)}$ \\ (1) Vicepresidencia Transmisión y Distribución Energía, Empresas Públicas de Medellín, Carrera 58 No. \\ 42-125, Medellín - Colombia (e-mail: carlos.garcia.montoya@epm.com.co) \\ (2) Departamento de Ingeniería Eléctrica, Universidad de Antioquia, Calle 67 No. 53-108, Medellín - \\ Colombia (e-mail: jmaria.lopez@udea.edu.co)
}

Recibido Ago. 2, 2016; Aceptado Oct. 6, 2016; Versión final Nov. 25, 2016, Publicado Abr. 2017

\begin{abstract}
Resumen
Se han caracterizado los costos de distribución de energía eléctrica incluyendo un indicador de calidad del servicio relacionado con la frecuencia de interrupciones en el modelamiento de los mismos. Inicialmente se postulan variables explicativas de costos y se utiliza un modelo de regresión multivariada para evaluar la capacidad explicativa de tales variables. Por otro lado, se emplean modelos de fronteras de eficiencia estocástica para determinar el nivel de eficiencia de las empresas que pertenecen a la muestra. Mediante el uso de estas metodologías se logró obtener tres modelos de regresión que permiten hacer una estimación de costos medios en los cuales incurren los distribuidores. Por medio de fronteras de eficiencia, se logró determinar otros tres modelos que permiten estimar la eficiencia de las empresas, pudiendo además identificar variaciones en su eficiencia según el modelo empleado.
\end{abstract}

Palabras clave: modelos de fronteras de eficiencia; distribución de energía eléctrica; análisis de fronteras estocásticas, calidad del servicio

\section{Characterization of the Cost of Electricity Distribution by means of Efficiency Frontier Models considering a Quality Service Index}

\begin{abstract}
Cost characterization of electricity distribution including a quality service index related to the interruption frequency in the modelling of electricity distribution costs has been done. Initially, explanatory variables of costs are postulated and a multivariate regression model is used to evaluate the explanatory capability of such variables. Furthermore, stochastic frontier models are used to determine the eficiency level of the companies belonging to the sample. By using these methodologies it was possible to obtain three regression models that allow estimating the average costs incurred by distribution companies. By means of efficiency frontiers it was possible to detemine other three models that allow estimating the efficiency of the companies, being also able to identify variations in their efficiency according to the model used.
\end{abstract}

Keywords: efficiency forntier models; electric power distribution; stochastic frontier analysis, quality of service 


\section{INTRODUCCIÓN}

La distribución de energía eléctrica es considerada un monopolio natural, dado que cumple con los elementos típicos que caracterizan a estos: economías de escala, subaditividad de costos, economía de alcance y barreras de entrada al mercado (Arias et al., 2004). Dada esta situación, la distribución de energía eléctrica es sometida a constante escrutinio por parte de los entes reguladores, los cuales, obviamente, buscan obtener la máxima eficiencia de las empresas que desarrollan esta actividad. Dentro de los diferentes aspectos que afectan e importan, tanto al regulador como a los demás actores del mercado, se encuentra la mejora de la calidad del servicio, la cual bajo condiciones de monopolio se puede ver descuidada por parte de las empresas de distribución. Dentro del negocio de la distribución de energía eléctrica la calidad del servicio es considerada como uno de los aspectos claves debido al efecto sobre la percepción del cliente y el impacto económico que éste puede tener cuando desmejora (Alfonso et al., 2007; Gómez et al., 2012). En (Küfeoğlu, et al., 2016) se efectúa una revisión de las metodologías empleadas tanto para la estimación del valor del costo de confiabilidad, así como de las técnicas de evaluación de costos de interrupción de cara al cliente. Este trabajo cubre una revisión entre 1990 y 2015. Por otro lado, en (Corton, et al., 2016) se presenta un estudio enfocado en el objetivo de identificar el impacto de las mejoras en calidad sobre los costos operativos; todo esto bajo el entorno regulatorio de Brasil y su esquema regulatorio particular. Por otro lado, desde el enfoque de modelamiento econométrico, en (Saastamoinen, et al., 2016) se presenta un estudio que aplica métodos de análisis de productividad en la determinación de un modelo de frontera que considera la calidad del servicio, específicamente mediante un método de estimación semi no paramétrico, bajo el objetivo primordial de definir los objetivos de calidad de las empresas de un sector. El caso de estudio es analizado dentro del mercado eléctrico de Finlandia. Estos trabajos muestran la continua necesidad que tiene el sector eléctrico, en diferentes países, de lograr un modelamiento adecuado de los límites de calidad logrables por las empresas y el efecto en los costos de distribuir energía. Finalmente, bajo este contexto, los entes reguladores hacen una constante revisión de los diferentes aspectos que afectan la distribución y su mejoramiento eficiente simulando las condiciones de competitividad.

La calidad del servicio de las empresas de distribución ha sido modelada o caracterizada desde diferentes ópticas, unas en busca de valores de eficiencia que le permitan a las empresas lograr niveles de calidad acorde con los mercados que atiende y otras con objetivos regulatorios que buscan lograr niveles de costo eficiencia adecuados al valor de la remuneración; además, buscando incentivar a las empresas en la mejora de eficiencia y calidad del servicio (Dimitrios et al., 2005; Saastamoinen, et al., 2016). Desde otro ángulo, se han desarrollado investigaciones que permiten identificar el efecto de las variables ambientales o del entorno sobre la calidad del servicio (Growitsch et al., 2010). Otra necesidad surge al enfocarse en la estimación del costo de la calidad del servicio en función de algunas variables características que permiten el modelamiento de este aspecto bajo metodologías econométricas (Jamasb et al., 2012).

Tanto desde el punto de vista de la regulación como desde el punto de vista del distribuidor, es conveniente conocer si los modelos empleados para caracterizar o modelar los costos del distribuidor se ven representados en términos de variables relacionadas directamente con el reconocimiento o el ingreso. La empresa de distribución está interesada directamente en la estimación de costos eficientes que le permitan determinar los límites de eficiencia en costos que le sean posibles alcanzar en un año o en un periodo regulatorio. Por otra parte, es el agente regulador quien se interesa por definir la senda de reducción de costos o reducir la brecha entre la práctica o frontera de costos que representa al mercado y la realidad de cada una de las empresas. Bajo ambos escenarios se evidencia la necesidad de contar con un modelo que, en términos generales, caracterice los costos de las empresas y defina las posibles mejoras o brechas, para con ello lograr una operación más eficiente de las empresas y en general, la eficiencia del sector como un todo.

El modelamiento de los costos de las empresas de distribución, con miras a la búsqueda de una mejor eficiencia, se ha desarrollado de manera general mediante técnicas de frontera de eficiencia (Patiño et al., 2010). Estas técnicas permiten identificar el peso de cada variable explicativa sobre el resultado económico. Desde este mismo ángulo, la caracterización del costo de distribución de energía eléctrica, considerando indicadores de calidad del servicio, puede dar señales a los actores del negocio sobre la eficiencia de las empresas y el impacto en los costos de las variables que miden la calidad del servicio. Lo anterior permite a las empresas realizar una estimación de sus costos eficientes considerando la calidad del servicio que pueden brindar. Si bien existen trabajos que modelan o caracterizan los costos de distribución considerando variables de entorno que afectan el desempeño general de las empresas (Growitsch et al., 2010); la principal contribución de éste artículo radica en mostrar que puede determinarse una función de producción que caracterice los costos de la distribución y permita validar el efecto de indicadores de calidad sobre los costos. Adicionalmente, se considera dentro del modelamiento la energía distribuida o vendida por el distribuidor, con miras a estimar costos eficientes frente a posibles cambios regulatorios que afecten el ingreso y los costos de las empresas. Esto permite que se realicen estimaciones del impacto debido a cambios en los mecanismos regulatorios implementados. 
En el presente trabajo se emplea una metodología de estimación de fronteras de eficiencia paramétricas para modelar el negocio de la distribución. Esta metodología incorpora un indicador de calidad del servicio, en este caso el denominado SAIFI (por sus siglas en inglés: System Average Interruption Frequency Index). Este indicador permite medir la frecuencia promedio de interrupciones percibida por los clientes de un sistema de distribución o una empresa en particular. Previo a esta estimación, mediante una regresión multivariada se valida la capacidad de explicación de costos de las variables disponibles para la estimación del modelo econométrico. Finalmente, se logra la estimación de tres modelos de frontera de eficiencia paramétricos que permiten identificar el efecto de las variables características del mercado atendido por el distribuidor de energía, como son: longitud de las redes, clientes atendidos, energía transmitida por el sistema y, como ya se mencionó, un indicador de calidad del servicio, con lo cual se logra la caracterización de los costos considerando la calidad del servicio.

\section{ESTIMACIÓN DE MODELOS DE FRONTERAS DE EFICIENCIA}

Para estimar los modelos de frontera de eficiencia que consideran la calidad de servicio se realizó una exploración inicial de variables postuladas como posibles explicativas de costo, con ello se lograron tres modelos de regresión. El posterior modelamiento de los costos de distribución se hizo mediante modelos de frontera de eficiencia aplicado a las variables explicativas más representativas. De este proceso se obtuvieron tres modelos, el modelo uno (modelo 1) considera cuatro variables: energía transmitida, kilómetros de red, clientes atendidos y el SAIFI. El segundo modelo (modelo 2) no involucra la energía transmitida y el tercer modelo (modelo 3) que no considera la energía transmitida pero si el SAIFI. Se hace este análisis considerando la posibilidad de que la remuneración del distribuidor se realice mediante Price Cap (Precio máximo) o mediante Revenue Cap (Ingreso máximo). Estas formas de regulación determinan un precio máximo por unidad de energía vendida (Price Cap), y un ingreso máximo para un periodo de tiempo que generalmente es de un año (Revenue Cap). En el primer tipo de regulación se traslada la incertidumbre del crecimiento o decrecimiento de la demanda al operador de red, mientras en el segundo se le garantiza un ingreso máximo al operador y no se le traslada el riesgo de efecto en el ingreso por variaciones de la demanda. El análisis realizado sobre los modelos obtenidos permite validar si estos son aplicables en ambos mecanismos regulatorios, permitiendo definir los costos eficientes de un conjunto de empresas considerando la incertidumbre o no que cada una tiene implícita según el mecanismo utilizado.

\section{Medición de Eficiencia y Productividad Mediante Fronteras de Eficiencia}

Desde el punto de vista económico se ha desarrollado toda una teoría relacionada con la medición de eficiencia y productividad, la cual es empleada por los agentes reguladores para determinar la eficiencia de las empresas, dentro de ellas, las del sector eléctrico. La productividad de una empresa se define como el coeficiente entre la cantidad de producto obtenido y el monto de los recursos empleados para la producción. El resultado obtenido se ve influenciado por la tecnología de producción y algunos agentes exógenos que afectan el proceso. De esta forma se llega a la eficiencia productiva, que corresponde a la capacidad de la empresa de producir a un costo determinado. Desde el punto de vista microeconómico existen dos tipos de eficiencia: la eficiencia técnica y la asignativa. La eficiencia técnica se define como la habilidad de obtener máximo producto bajo unos niveles definidos de insumos y tecnología de producción, midiendo la habilidad de usar bajos recursos para producir un número de productos determinado. Desde otro ángulo, la eficiencia asignativa es la habilidad de combinar de manera óptima los productos e insumos disponibles (Sanhueza, 2007; Pahwa et al., 2003). El nivel óptimo de eficiencia puede definirse como el costo u otro objetivo que la compañía tenga definido. De esta forma surgen dos tipos de modelos, clasificados de manera general como modelos de frontera de costos y de producción (Melo et al., 2005).

La medición de eficiencia respecto a modelos de frontera de costos permite medir la eficiencia económica. Esto se hace evaluando la diferencia entre los costos empleados en la producción y los costos del modelo óptimo. De manera ilustrativa, puede apreciarse en la Fig. 1. a), las observaciones denominadas $\mathrm{C}$ y $\mathrm{B}$ son ineficientes económicamente, mientras la observación A por encontrarse sobre la frontera es considerada una observación eficiente. En la Fig. 1. a) la variable del eje de las abscisas (identificado como Y) corresponde a las unidades producidas por las empresas y el eje de las ordenadas corresponde a los costos en que se incurre para la producción. En la Fig. 1. b), a diferencia de la anterior, el eje de las abscisas corresponde a las entradas o insumos requeridos para la producción y el eje de las ordenadas corresponde a las unidades producidas o salidas del sistema. Las medidas de eficiencia respecto a fronteras de producción, permiten lograr una medición de la eficiencia técnica, respecto al número de productos máximo que pueden obtenerse dado un conjunto de insumos del proceso de producción (Melo et al., 2005). Esto se interpreta como la capacidad de mejora de producción que posee una unidad productiva respecto a la frontera. En la Fig. 1. b), puede apreciarse como la medición $B$ posee un margen de mejora respecto a la medición $A$, lo cual permite identificar qué $A$ es más eficiente técnicamente que $B$. 


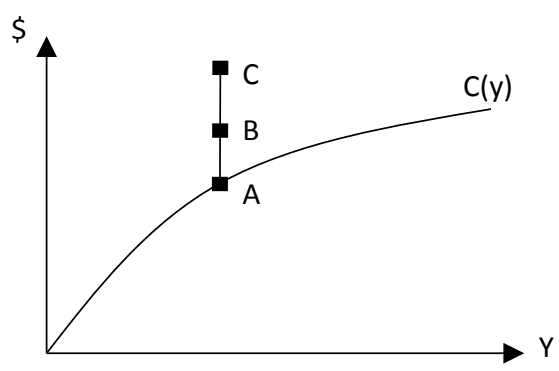

a) Fronteras de Costo

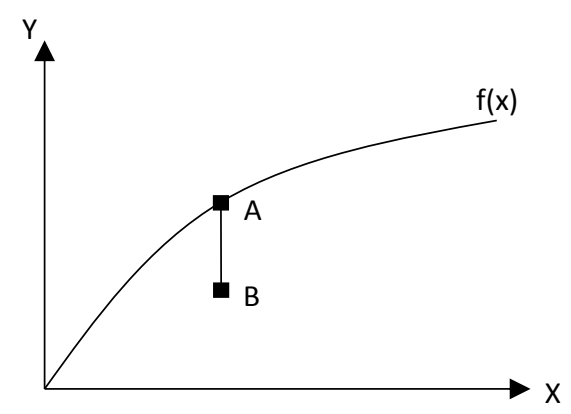

b) Fronteras de Producción

Fig. 1: Medidas de Eficiencia en Fronteras

De manera general, se han desarrollado dos metodologías para la estimación de los modelos de frontera que se han mencionado: primero el denominado análisis envolvente de datos (DEA por su sigla en inglés) cuya estimación está basada en programación lineal de forma no paramétrica y, segundo, el denominado análisis de fronteras estocásticas (SFA por su sigla en inglés) que está fundamentado en métodos econométricos con su respectiva forma funcional que lo convierte en un modelo paramétrico. Estos métodos de medición de eficiencia se han llevado a diversas aplicaciones, pero especialmente han tenido éxito al ser aplicadas a la regulación de mercados monopólicos y en los benchmark entre empresas del sector. Su potencial radica en que hacen posible comparar un grupo de empresas con mercados y características diferentes, permitiendo una regulación o comparación equitativa para las mismas (Rodríguez et al., 1999). En los últimos años han surgido técnicas híbridas que permiten integrar las ventajas de las técnicas paramétricas y no paramétricas como la propuesta en (Kuosmanen et al., 2013). En este artículo se utiliza el análisis de fronteras de eficiencia. Esta metodología fue elegida por el enfoque paramétrico que permite identificar el efecto que tienen las variables postuladas como explicativas sobre los costos de las empresas de distribución y por su versatilidad, pues permite realizar análisis de sensibilidad sobre el efecto de diferentes variables y proponer diferentes modelos.

\section{Análisis de Fronteras de Eficiencia}

La estimación de fronteras de eficiencia se ve determinada en principio por la forma funcional que debe ser propuesta. De manera general, la forma funcional contiene dos componentes principales: el determinístico y el estocástico. La ecuación (1) presenta la forma general de un modelo paramétrico estimado mediante fronteras de eficiencia.

$$
y=f(x)+v-u
$$

El componente determinístico, representado por $f(x)$, representa la forma funcional que toman los insumos del modelo econométrico, $y$ corresponde al producto en el caso de las funciones de producción y a los costos en el caso de las fronteras de costo, $v-u$ corresponden a la componente estocástica del modelo, el cual permite separar el término del error $v$ que captura las perturbaciones o ruido del término $u$ que representa la ineficiencia técnica de las empresas que componen la muestra. El componente determinístico de la frontera puede tomar diferentes formas dependiendo del modelo de producción propuesto. Las formas funcionales más comunes dentro de las fronteras de eficiencia son la Cobb-Douglas y la Translogarítmica. En el presente trabajo se emplea la función de producción Cobb-Douglas, la cual se representa en la ecuación (2). Donde $Y_{i}$ corresponde a los costos de la empresa $i$ y $X_{n i}$ corresponde a la variable $n$ de la empresa $i$. Por su parte los $\beta$ corresponden a los coeficientes que determinan el peso de la variable en la frontera. Para mayores detalles sobre la metodología de estimación puede consultarse (Kumbhakar et al., 2000).

$$
\operatorname{Ln} Y_{i}=\beta_{0}+\sum_{n=1}^{N} \beta_{n} \operatorname{Ln} X_{n i}+v_{i}+u_{i}
$$

Una vez se cuenta con una forma funcional propuesta para la estimación de la frontera de eficiencia debe seleccionarse un grupo de variables que permitan representar o caracterizar el negocio de la distribución. De manera general estas variables se clasifican en insumos y productos o en entradas y salidas. Para el caso de la distribución de energía en la literatura se han propuesto gran cantidad de variables, entre ellas: número de 
clientes atendidos, kilómetros de redes de distribución, $\mathrm{km} 2$ de área atendida, energía vendida en $\mathrm{kW} / \mathrm{h}$ año o GW/h año, variables ambientales, entre otras (Patiño et al., 2010). A pesar de existir muchas variables consideradas características de la distribución de energía eléctrica, no se ha encontrado un consenso respecto a conjunto único de variables como las más representativas y son escasos los trabajos que involucran como variable explicativa algún indicador o índice que esté relacionado con la calidad del servicio. En el presente trabajo se proponen como variables explicativas a considerar: el número de clientes atendidos, los kilómetros de red del sistema de distribución, energía vendida (GW/h año), el indicador de calidad del servicio SAIFI, y como variable explicada se determinó el costo AOM total del distribuidor (Administración, Operación y Mantenimiento). Estas fueron elegidas dentro de las propuestas en otros trabajos considerando su importancia y peso explicativo dentro de la frontera, además de su capacidad descriptiva en términos de dimensión de infraestructura, densidad y tamaño del mercado atendido por el distribuidor. A pesar de que al inicio fue postulado el SAIDI como variable explicativa, esta fue retirada dado que presentó un muy bajo peso explicativo en la exploración inicial mediante modelos de regresión multivariada. Por otra parte, dada su importancia para el negocio de distribución de energía, la variable pérdidas de energía podría considerarse explicativa. Si bien esta es una variable relevante para el negocio de distribución, no se cuenta con una medición homologada de esta variable, de modo tal que garantice un resultado adecuado y confiable al estimar los modelos de regresión y de frontera. Finalmente, para la estimación del modelo se empleó el paquete estadístico STATA y para el ajuste del modelo econométrico se empleó una muestra transversal que consta de 30 observaciones de empresas colombianas, lo cual, para el número de variables explicativas empleadas es coherente estadísticamente. En la Fig. 2 se ilustra un esquema general de la metodología con el objetivo de facilitar la comprensión del procedimiento desarrollado.

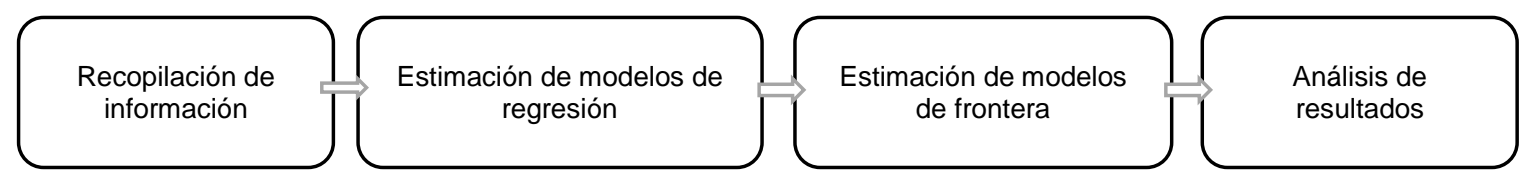

Fig. 2: Esquema general de la metodología empleada.

\section{RESULTADOS Y DISCUSIÓN}

Como se mencionó en la sección anterior, la muestra empleada está comprendida por 30 observaciones de empresas colombianas. En la Tabla 1 se presenta un resumen de la estadística descriptiva relacionada con las variables consideradas. Note que la muestra es ampliamente variable en cuanto a dimensión de las empresas (infraestructura representada en kilómetros de red y de clientes atendidos por el sistema). Se registran empresas que atienden entre 155,127 clientes y 4,069,259 clientes; con respecto a la infraestructura se tienen valores entre 4,812 kilómetros de red y 85,379 kilómetros de red. Finalmente, en área de cobertura se aprecia que las empresas tienen entre 1,962 kilómetros cuadrados y 60,728 kilómetros cuadrados. Lo anterior evidencia la diversidad de las empresas en términos de ruralidad y alta concentración urbana, lo cual, dada la naturaleza del negocio de la distribución puede representar economías de escala en su operación.

Tabla 1: Estadística descriptiva de la muestra empleada.

\begin{tabular}{|l|l|l|r|r|r|r|}
\hline \multicolumn{1}{|c|}{ Descripción } & \multicolumn{1}{|c|}{ Variable } & \multicolumn{1}{c|}{ Unidad } & Media & $\begin{array}{c}\text { Desv. } \\
\text { estándar }\end{array}$ & \multicolumn{1}{c|}{ Míni } & Máx \\
\hline Costo total de distribución & Costo & Millones de COP & 148,097 & 98,500 & 35,146 & 348,619 \\
\hline Empleados & Emp & Número & 844 & 333 & 327 & 1,291 \\
\hline Área de cobertura & Área_C & Kilómetros cuadrados & 25,060 & 20,726 & 1,962 & 60,728 \\
\hline Energía transportada por el sistema & Energ_tran & GWh & 228 & 252 & 30 & 786 \\
\hline Clientes & Clien & Número & $1,018,538$ & $1,295,952$ & 155,127 & $4,069,259$ \\
\hline Kilómetros de red & Kms_Total & Kilómetros & 33,818 & 25,247 & 4,812 & 85,397 \\
\hline Frecuencia media de interrupción & SAIFI & Interrupciones & 34.68 & 17.53 & 7.94 & 40.53 \\
\hline
\end{tabular}

Con el objetivo de validar la capacidad explicativa de las variables sobre los costos de distribución incluyendo las variables que se han mencionado desde el inicio, se procede a la ejecución de tres regresiones lineales multivariadas. La regresión 1 obtuvo un valor de $\mathrm{R}^{2}$ de 0.979 (coeficiente de determinación), la regresión 2 un $\mathrm{R}^{2}$ de 0.977 y la tercera un $\mathrm{R}^{2}$ de 0.978 , cumpliendo con un valor de ajuste cercano a 1 , lo cual indica que son modelos con ajuste adecuado y que las variables explican adecuadamente el comportamiento de los costos. En la Tabla 2 se presentan los resultados generales de las tres regresiones realizadas y sus respectivos coeficientes. 
Tabla 2: Parámetros estimados y datos de los modelos de regresión estimados.

\begin{tabular}{|l|l|c|c|c|c|c|c|}
\hline \multirow{2}{*}{ Variable } & \multirow{2}{*}{ Unidad } & \multicolumn{2}{c|}{ Regresión 1 } & \multicolumn{2}{c|}{ Regresión 2 } & \multicolumn{2}{c|}{ Regresión 3 } \\
\cline { 3 - 8 } & & Coeficiente & Error standard & Coeficiente & Error standard & Coeficiente & $\begin{array}{c}\text { Error } \\
\text { standard }\end{array}$ \\
\hline Ln Energ_tran & GWh & 0.0814 & 0.0574 & No incluida & - & 0.0635 & 0.0555 \\
\hline Ln Clien & Número & 0.2119 & 0.0921 & 0.2447 & 0.0913 & 0.1404 & 0.0669 \\
\hline Ln Kms_Total & Kilómetros & 0.4926 & 0.0756 & 0.5274 & 0.0732 & 0.5485 & 0.0572 \\
\hline Ln SAIFI & Interrupciones & 0.1517 & 0.1352 & 0.0985 & 0.1330 & $\begin{array}{c}\text { No } \\
\text { incluida }\end{array}$ & - \\
\hline Constante & - & 3.0230 & 0.8961 & 2.8051 & 0.9970 & 3.9741 & 0.5064 \\
\hline
\end{tabular}

Estos resultados confirman que las variables de energía transportada y SAIFI pueden considerarse como variables explicativas de costos de las empresas de distribución involucradas en este modelo. Con el objetivo de evidenciar el efecto que tenga la inclusión de SAIFI y energía transportada sobre el modelo, tanto de manera conjunto como por separado, se efectuaron las tres regresiones ya mencionadas. La primera regresión incluyendo todas las variables, la segunda excluyendo la energía transportada y la tercera excluyendo el SAIFI. Esto permite identificar el efecto sobre el modelo de costos que tienen estas variables al ser incluidas y retiradas del mismo. Como puede apreciarse el $\mathrm{R}^{2}$ de las regresiones no cambia significativamente con las variantes que se le imprimieron al modelo. Por otra parte, si se detallan los coeficientes podemos observar en la regresión 1 que el SAIFI tiene un coeficiente con un valor casi del doble del correspondiente a la energía transportada. Esto indica que su efecto sobre los costos es superior. Al no incluir la variable de energía en la regresión 2 se evidencia una leve reducción en el coeficiente del SAIFI, mientras que en la regresión 3 se aprecia un efecto similar sobre la variable energía transportada al no considerar el SAIFI. A pesar de presentar variaciones por la inclusión o no de las variables mencionadas, los ajustes observados sobre los coeficientes no son de magnitudes considerables ni presentan cambios de signo, con lo cual los modelos se presentan consistentes ante las variaciones.

Luego de confirmada la aplicabilidad sobre modelos de costos de distribución empleando las variables y los modelos propuestos, se procede a la estimación de las fronteras de eficiencia empleando estas mismas variables. En la Tabla 3 puede apreciarse los parámetros obtenidos de los modelos propuestos.

Tabla 3: Parámetros estimados y datos de los modelos estimados.

\begin{tabular}{|l|l|c|c|c|c|c|c|}
\hline \multirow{2}{*}{ Variable } & \multirow{2}{*}{ Unidad } & \multicolumn{2}{c|}{ Modelo 1 } & \multicolumn{2}{c|}{ Modelo 2 } & \multicolumn{2}{c|}{ Modelo 3 } \\
\cline { 3 - 8 } & & Coeficiente & Error standard & Coeficiente & Error standard & Coeficiente & $\begin{array}{c}\text { Error } \\
\text { standard }\end{array}$ \\
\hline Ln Energ_tran & GWh & 0.0291 & 0.0512 & No incluida & - & 0.0225 & 0.0494 \\
\hline Ln Clien & Número & 0.3035 & 0.1035 & 0.3349 & 0.0921 & 0.2141 & 0.0756 \\
\hline Ln Kms_Total & Kilómetros & 0.4565 & 0.0712 & 0.4497 & 0.0697 & 0.5193 & 0.0529 \\
\hline Ln SAIFI & Interrupciones & 0.1630 & 0.1355 & 0.1548 & 0.1391 & No incluida & - \\
\hline Constante & - & 2.2747 & 1.0714 & 2.0892 & 1.0441 & 3.3762 & 0.4904 \\
\hline
\end{tabular}

Los modelos de regresión presentados en la Tabla 2 fueron la base para ejecutar los modelos de frontera de eficiencia de costo que se presentan en la Tabla 3. El ejercicio de inclusión y retiro de las variables fue similar. Ahora, en el caso de los modelos de frontera, se pretende identificar las variaciones tanto desde los resultados generales de los modelos de frontera como de los resultados de eficiencia de las empresas y los posibles cambios que se presenten con la variación de los tres modelos. Todo esto, prestando especial atención al efecto de la inclusión y exclusión de las variables que se han mencionado. Es así como puede apreciarse que las modificaciones efectuadas, en los modelos 2 y 3 , no generan variaciones muy significativas sobre los coeficientes de los mismos, asunto que se refleja de manera similar en los resultados del error estándar de cada variable. Esta situación permite confirmar la consistencia de los modelos de frontera ajustados con las variables propuestas, para con ello proceder con la estimación de la eficiencia de las empresas frente a las fronteras logradas.

Una vez logrados los modelos de frontera de eficiencia que caracterizan el grupo de empresas bajo análisis, fueron calculados los niveles de eficiencia de las mismas frente a cada una de las fronteras. Adicionalmente, se calculó el promedio de eficiencia para cada empresa, esto con el objetivo de identificar las posibles variaciones de magnitud de la eficiencia frente a cada una de las fronteras. En la Fig. 3 se presenta la comparación de los resultados de medición de eficiencia de las empresas que componen la muestra para los tres modelos de frontera y el promedio de eficiencia calculado. 
Puede apreciarse en la Fig. 3, como algunas empresas mejoran o desmejoran en eficiencia dependiendo del modelo contra el cual se efectúa la medida de eficiencia. Esto permite identificar la sensibilidad que puede tener, para una u otra empresa, el hecho de ser medida incluyendo diferentes variables e igualmente el efecto del cambio en la metodología regulatoria, en caso de considerar el cambio de variables explicativas de costo. Algunas empresas, como las identificadas como 15 y 29 , a pesar de no mostrar un cambio sustancial respecto a la medición de eficiencia frente a los tres modelos, si presentan una mejor eficiencia cuando esta es medida frente al modelo 2. Sin embargo, dado que la diferencia en medición no es superior a un $3 \%$ en eficiencia, el efecto se considera aceptable al comparar los resultados de los modelos. Adicionalmente, si se calcula la variación promedio de la eficiencia entre los resultados de los modelos, esta llega a un valor de $0.54 \%$, el cual es un valor que puede considerarse bajo a pesar de los ajustes de los modelos realizados. Dado que se presentan variaciones pequeñas, los resultados entre los modelos pueden considerarse consistentes, lo cual permite verificar gráficamente el efecto que tiene el incluir o no la variable energía vendida y SAIFI dentro del modelo.

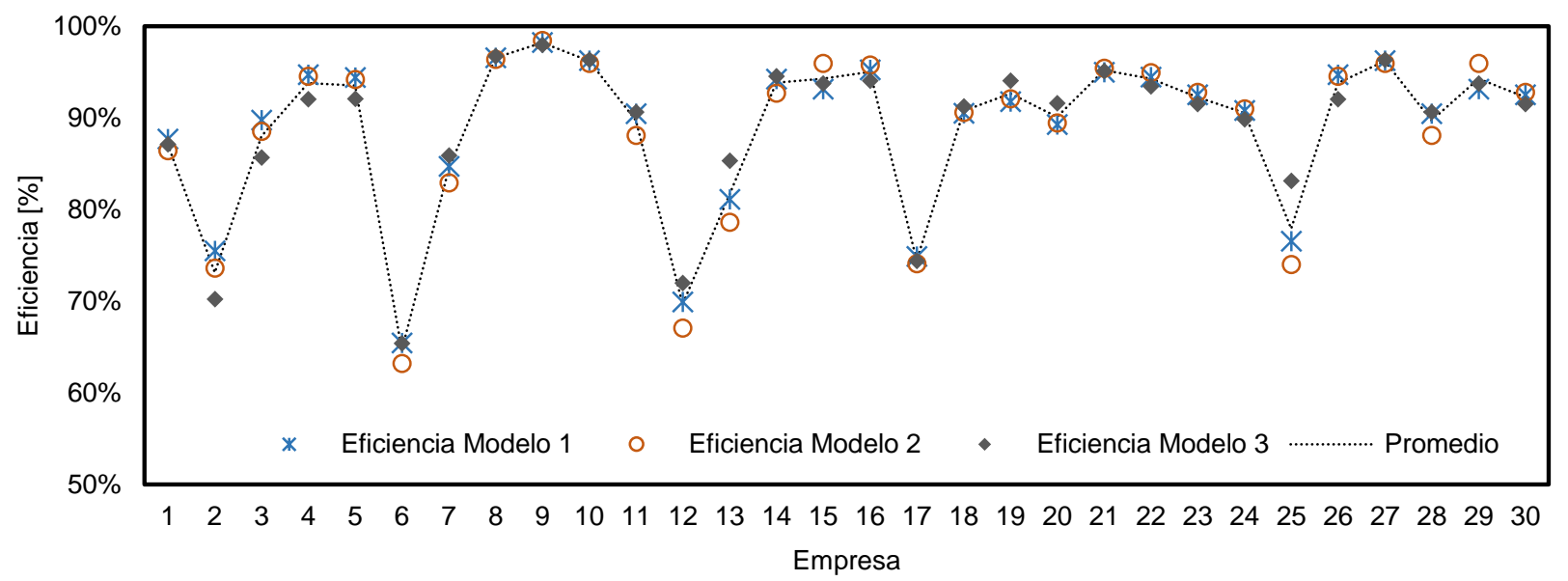

Fig. 3: Eficiencia calculada para la muestra completa y la media de eficiencia que se observa en las empresas.

Los resultados logrados han permitido validar que es posible modelar econométricamente los costos de distribución mediante un grupo de variables características del negocio, incluyendo entre ellas la energía vendida o distribuida y, especialmente, un indicador de calidad del servicio como variable explicativa. Los modelos obtenidos permiten identificar el efecto que tiene sobre los costos de distribución el indicador de calidad del servicio, logrando de esta forma cuantificar el costo de alcanzar un valor específico de calidad y su efecto conjunto con otras variables, como las presentes en los modelo desarrollados. Ahora, al observar el coeficiente del SAIFI en los modelos que lo incluyen, se puede observar un efecto mayor sobre la frontera que el valor presente por la energía vendida, dado el mayor valor que presenta el coeficiente asociado al SAIFI. Esta situación tiene implicaciones de cara a su aplicación en la regulación, u otras aplicaciones, dado que el costo de distribución definido bajo los modelos que incluyen el SAIFI sería más susceptible a cambios de esta variable. Por otra parte, el efecto que se observa de la variable energía transmitida sobre la estimación de la frontera de costos es mucho menor al compararlo con el efecto del SAIFI, lo que lleva a pensar que esta pueda ser retirada fácilmente sin un efecto mayor, obviamente dependiendo de la aplicación que se dé al modelo. Por último, la variable explicativa que mayor peso representa en los modelos es la correspondiente a los kilómetros de red. Esto tiene una alta coherencia con la dinámica del negocio, dado que tanto los costos operativos como los costos de capital están altamente relacionados con las redes que se requieren para atender un mercado o región en especial.

Como se explicó con anterioridad, uno de los objetivos de involucrar las variables que se han empleado es lograr modelos que se adapten a dos mecanismos regulatorios diferentes como son Price Cap y Revenue Cap, permitiendo la diferenciación de la energía transmitida como variable con una alta incertidumbre y la correspondiente calidad del servicio que las empresas brindan con unos recursos estimados. Observando los resultados de cara a su aplicabilidad, bien sea para estimación de costos eficientes o para medir la eficiencia de las empresas, se puede afirmar que el efecto de la variable energía transmitida, como ya fue mencionado, no es muy notable, cosa diferente sucede con el SAIFI. Esto permite identificar que, al momento de elegir un modelo adecuado para alguno de los mecanismos regulatorios debe considerarse inicialmente cuales son las variables que se desea tener definidas o estimadas mediante el modelo. Es así como se puede recomendar entre los modelos 1 y 3 para un mecanismo regulatorio Price Cap, que además incluya la búsqueda de costos eficientes, dado que al estimar la energía transmitida dentro del modelo ésta ya estaría considerada para las estimaciones de la remuneración o la estimación de costos. De esta forma, se logra que al momento de la aplicación del mecanismo regulatorio se disminuya la incertidumbre implícita en el proceso, brindando valores estimados para 
la energía transmitida desde el momento de estimar los costos eficientes. Por otro lado, con respecto al Revenue Cap, los modelos 1 y 2 podrían ser adecuados. Sin embargo, dado que este no requiere de estimaciones de energía transmitida, se podría dar una más adecuada estimación al emplearse el modelo 2.

La metodología descrita anteriormente se implementó con el objetivo de cuantificar la variación de la eficiencia de las empresas de distribución ante diferentes modelos, y estimar sus costos bajo diferentes mecanismos regulatorios, considerando un indicador de calidad del servicio. El modelo que se emplee sobre un caso real tendrá efecto directo sobre la remuneración. Por otro lado, desde el punto de vista tarifario puede afirmarse que el efecto sobre la tarifa es directamente proporcional a la magnitud de la remuneración calculada para las empresas. Sin embargo, un estudio sobre el esquema tarifario está fuera del alcance del presente artículo.

El considerar la calidad del servicio desde el modelo permite que la estimación se ajuste a valores reales de la calidad esperada, según los limitantes, endógenos o exógenos, de la empresa. Es así como puede definirse un nivel de costos eficiente para un grupo de empresas mediante los modelos logrados y, posteriormente, mediante mecanismos de ajuste (como incentivos y penalizaciones dentro de los esquemas regulatorios), puede reconocerse las mejoras o disminuciones en calidad del servicio logrado por las empresas. Esto permite que la estimación de costos eficientes defina un objetivo de costos y los mecanismos de incentivo o ajuste puedan reconocer las variaciones.

\section{CONCLUSIONES}

Los resultados logrados han permitido validar que es posible modelar econométricamente los costos de distribución mediante un grupo de variables características del negocio, incluyendo entre ellas la energía transmitida y un indicador de calidad del servicio como variable explicativa.

Se desarrollaron tres modelos de fronteras que se ajustan a diferentes necesidades y entornos, tanto desde el punto de vista de las empresas como desde la mirada de los mecanismos regulatorios.

Los modelos desarrollados mediante SFA representan de manera adecuada y consistente los costos y la medición de eficiencia de las empresas, frente a las fronteras de costos estimadas.

Para la selección y aplicación de alguno de los modelos propuestos, se debe tener en cuenta que las variables explicativas empleadas permitan hacer estimaciones con una precisión adecuada y acorde a cada caso. Esto es, en lo posible, elegir modelos con variables que representen de buena manera el mercado, esto facilitará la interpretación de las estimaciones y la estimación, tal como se presentó en este trabajo.

En las pruebas realizadas se pudo observar que el efecto de la variable energía transmitida sobre los resultados de los modelos es menor. Cosa que no sucede con el SAIFI, el cual posee un coeficiente que le hace más representativo. El uso en la estimación de costos de modelos como los logrados permite que se haga una estimación de costo eficiente acorde con las variables que caracterizan el mercado.

\section{AGRADECIMIENTOS}

Los autores agradecen a la Universidad de Antioquia (UdeA) por el apoyo del proyecto "Sostenibilidad 20162017" para el desarrollo de este trabajo.

\section{REFERENCIAS}

Alfonso, J. L., Batista, J., Sepúlveda, M. J., \& Martins, J. S., Sistema digital de bajo coste para la monitorización de la calidad de energía eléctrica, Información Tecnológica, 18(4), 15-23 (2007)

Arias, E., C. Enrique, H. Cadavid y V. José, La Regulación Económica de la Distribución de la Energía Eléctrica, Ecos de Economía, 8(18), 99-139 (2004)

Corton, M. L., Zimmermann, A., Phillips, M. A., The low cost of quality improvements in the electricity distribution sector of Brazil, Energy Policy, 97, 485-493 (2016)

Dimitrios G., T. Jamasb, M. Pollitt, Benchmarking and incentive regulation of quality of service: an application to the UK electricity distribution networks, Energy Policy, 33(17), 2256-2271 (2005)

Gómez, V. A., Peña, R. A., y Hernández, C., Identificación y Localización de Fallas en Sistemas de Distribución con Medidores de Calidad del Servicio de Energía Eléctrica, Información Tecnológica, 23(2), 109-116 (2012) 
Growitsch, C., T. Jamasb and H. Wetzel, Efficiency effects of quality of service and environmental factors: experience from Norwegian electricity distribution (en línea), Acceso: 12 de Julio (2016) http://www.econ.cam.ac.uk/dae/repec/cam/pdf (2010)

Jamasb T., L. Orea y M. Pollitt, Estimating the marginal cost of quality improvements: The case of the UK electricity distribution companies, Energy Economics, 34(5), 1498-1506 (2012)

Kumbhakar, S. y K. Lovell, Stochastic Frontier Analysis, Cambridge University Press, (2000)

Kuosmanen, T., Saastamoinen, A. y Sipilainen, T., What is the best practice for benchmark regulation of electricity distribution? Comparison of DEA, SFA and StoNED Methods, Energy Policy, 61, 740-750 (2013)

Melo, B. y N. Espinoza, Ineficiencia en la distribución de energía eléctrica: Una aplicación de las funciones de distancia estocástica, Revista ESPE, (49), 88-132 (2005)

Pahwa, A., F. Xiaoming y D. Lubkeman, Performance evaluation of electric distribution utilities based on data envelopment analysis, IEEE Transactions on Power Systems, 17(3), 400-405 (2002)

Patiño, A., G. Gómez y E. Osorio, Evaluación del desempeño del sector de distribución de electricidad en Colombia: Una aplicación del análisis de frontera estocástica, Ensayos sobre Política Económica, 28(62) (2010)

Rodríguez, M., M. Rossi y C. Ruzzier, Fronteras de eficiencia en el sector de distribución de energía eléctrica: la experiencia sudamericana, Textos de Discusión №15, Instituto de Economía, Universidad Argentina de la Empresa (1999)

Saastamoinen, A. y T. Kuosmanen, Quality frontier of electricity distribution: Supply security, best practices, and underground cabling in Finland, Energy Economics, http://dx.doi.org/10.1016/j.eneco.2014.04.016, 53, 281-292 (2016)

Sanhueza, R. y H. Rudnick, Frontier methodologies for the determination of efficiencies in distribution costs, Ingeniare, Revista Chilena de Ingeniería, 15(3), 220-226 (2007)

Küfeoğlu, S. y M. Lehtonen, A review on the theory of electric power reliability worth and customer interruption costs assessment techniques, $13^{\text {th }}$ International Conference on the European Energy Market (EEM), Porto, 1-6 (2016) 
